\title{
Raising Emotional Intelligence from the Mat How Mindful Yoga Accelerates and Deepens Training in Emotional Intelligence
}

\author{
William L. Weis, Ph.D. \\ Professor of Management \\ Seattle University \\ Darcy Stewart, LMHC, C-IAYT \\ Licensed Mental Health Counselor \\ IAYT Certified Viniyoga Instructor \& Yoga Therapist
}

\begin{abstract}
In 2003 we began teaching an innovative graduate course designed to help our MBA (Master of Business Administration) students at Seattle University develop their emotional intelligence skills. As of today, in early 2019, we have delivered the course, Leading with Emotional Intelligence, to over 1500 graduate students in 53 classes. Six years ago, in the summer of 2013, we began augmenting our emotional intelligence (EQ) course activities with a "mindful" yoga regimen. This article reviews the impact that adding yoga has had on our EQ training milieu, and looks at how both the pace and depth of EQ skill development have been enhanced in the six years since we made this important course revision. We close by offering suggestions for how to effectively integrate yoga practice in an EQ teaching curriculum, and offer our help to those who may be interested in taking this step.
\end{abstract}

Keywords: Emotional Intelligence, Yoga, Mindfulness, Leadership, Self-Awareness, Presence, Meditation

\section{A Brief History of EQ at Seattle University}

The start of our 16-year journey teaching emotional intelligence (EQ) began in the Italian Dolomites in the summer of 2003 when we first offered an experimental MBA course called Leading with Emotional Intelligence as part of a twoweek study tour to Italy. The course was a unique opportunity to hone EQ skills while exploring the spectacular Dolomites mountain range of northern Italy, and included a set of experiential activities that blended hiking with EQ training. Today, in the winter of 2019 , we look forward to leading our $17^{\text {th }}$ consecutive "EQ in the Dolomites" program in September, and are currently offering the $54^{\text {th }}$ iteration of the EQ course here in Seattle during our winter quarter. Leading with Emotional Intelligence has become the most popular elective course in the MBA program, and is the only business elective to draw widespread participation from every graduate program on campus.

In the summer of 2013 we added a yoga component to our mix of experiential exercises comprising our EQ curriculum (see Appendix 1 for a list of those program elements). In this article we look at the impact that adding yoga practice has had on our EQ course experience. We base this assessment both on formal and informal feedback we have received from our course participants, as well as on our personal observations of the heightened awareness and intentionality that our graduate students are now bringing to the EQ training milieu.

We begin our discussion with a summary of the EQ course structure, including a brief description of the core experiential components of the curriculum to which yoga practice was eventually added. We believe this will frame for the reader a context for anticipating the incremental benefits that yoga could bring to EQ training, and offer a better understanding of yoga's auspicious fit in our course over the past six years. We first look at the learning objectives that have informed the development of our course since its inception in 2003.

\section{Key Learning Objectives of Leading with Emotional Intelligence}

The formal learning objectives of our EQ course, both before and after adding yoga to the class activities, flow from the broader learning goals of our graduate programs at Seattle University. As such, they target knowledge and skill sets that are appropriate for professional development. An extended list of learning objectives can be found in Appendix 1. In brief, the key objectives for the course are:

1. To enhance both self- and social-awareness, and develop more effective self-management and relationship-management skills.

2. To develop capacity for more effective communication with colleagues and clients delivered with authenticity, selfunderstanding and integrity. 
3. To develop and hone skills for engaging and harnessing the neural limbic system ("fight or flight" response) to replace impulsive and automatic response patterns with conscious choice patterns.

The main focus of this article is on the role that yoga can serve in achieving these learning outcomes in a program explicitly offered to enhance students' emotional intelligence skills. We begin with a look at the main innovative components that comprised our pre-yoga EQ course, and argue that this landscape of experiential exercises creates fertile ground for adding a yogic pathway toward mindfulness.

\section{Innovative Experiential Aspects of the EQ Training Program}

While there are many course elements in addition to those outlined below, we believe the following five components of the program account for the major transformational outcomes from the experience:

Innovative Elements
Coaching Module
Autobiography Exercise
T-Group Training
Three-Day Immersion Experience

And, as of 2013:

Mindful Yoga Practice

\section{Related Outcomes}

Active listening, curiosity, caring, patience

Self-understanding, empathy, resiliency,

Awareness, transparency, clarity, presence

Connection, openness, integrity, mutual caring

Self-awareness, mindfulness, response-modulation

The first three of these elements are briefly discussed in Appendix 2, the fourth is the container (a 3-day residential retreat) in which these elements become most intensively practiced. Finally, Mindful Yoga, the latest addition to our mix of course activities and now occupying nearly twenty percent of our class time, is discussed in the balance of this article.

\section{The Role of Mindful Yoga in EQ Training}

Finally, we arrive at the primary focus of this article - the role of yoga in our EQ training program and its impact on the learning experience since its addition in the summer of 2013. For readers who practice yoga, or who have in the past, the linkage with EQ training as we have described it in this discussion and in the appendices may already be abundantly evident. Emotional intelligence is, after all, about a mindful and intentional approach to human interaction. It is about being present and self-aware, and about creating space between stimulus and response. It is about harnessing impulse when the amygdale delivers a powerful emotion to the "fight, flight or freeze" instinct, and providing time and space for an intentional choice, behavior and communication, rather than simply for an instinctual reaction.

We would describe our yoga experiences and sensations (especially based on a yoga variation that prioritizes connecting breath with movement) with the words that we routinely associate with emotional intelligence: awareness, presence, mindfulness, intentionality. Quieting the mind, slowing down, creating space, being present, being mindful, being intentional, listening to our bodies, being aware of our emotions - in practicing yoga as in acting with emotional intelligence, our foundational aspirations are much the same. And they complement each other.

Thus, as employed in the EQ course, a primary intention of including a yoga practice is to set a tone of mindfulness and create a supportive container for the interpersonal work to come. In addition, the practice itself is intended to engender the intra-personal work of developing greater focus of attention, increased self-awareness, and the ability to modulate the autonomous nervous system's response to stress. Finally, including yoga practice at the end of class and retreat sessions helps students integrate new practices and sometimes emotionally vulnerable material. End of day/class yoga provides a space of calm to help students contemplate and metabolize their course experiences.

\section{How We Use Yoga to Complement our EQ Training Regimen}

Our typical EQ course schedule involves four full-day class meetings on our campus, in addition to a three-day residential retreat at a nearby conference center, where students arrive on Friday morning and leave late on Sunday afternoon. All in all, our entire program comprises approximately fifty hours of class-contact time. The annual "Dolomites" section of the course, on the other hand, is entirely delivered over nine days and eight nights in a hotel and in hiking lodges in the Italian Alps, and utilizes approximately sixty hours of class time in EQ course exercises.

Our ideal for placing yoga into this schedule is to offer a one-hour yoga session at the end of each of our four oncampus meetings, and to begin and end each day of our three-day retreat with an hour-long session. In total that would involve nine yoga sessions, filling nearly twenty percent of our total class-contact time. On retreat we start each day Friday, Saturday and Sunday - with a yoga session, and end the days on Friday and Saturday with yoga. On Sunday 
afternoon we conclude our retreat program by 4:00 PM, usually with a twenty-minute guided meditation and Zen activity. We have occasionally included a short yoga practice in this ending piece.

The Dolomites program starts and ends every day with yoga, except for the two nights when we stay in a hiking lodge high in the mountains. In total we schedule twelve yoga sessions in our Dolomites program, twenty percent of the sixty total contact hours.

\section{Yoga for Tone Setting}

The EQ exercises that fill our three days on retreat (nine days in the Dolomites) create intensity and emotional impact, sometimes to the point of emotional fatigue. A committed mindset is essential to meet the challenges of these retreat days, as well as a willingness to be transparent, and a willingness to create and preserve a space of safety and acceptance for one's own and one's student colleagues' vulnerabilities. As such, one of the roles of our morning yoga is to set a tone of intentionality, of attentiveness, and of non-judgmental acceptance - a fairly large order for that one hour in yoga practice. As inferred in our previous references to our practice, we follow what one might describe as a "slow flow" and "slow breath" yoga practice, where we flow seamlessly from one posture to another, staying connected to the breath with each movement, as well as with the transitions from one yogic posture platform to the next. Our flows are punctuated by periodic "stays" for short intervals involving four or five full breaths. With the exception of these "stays" and our final resting pose, we are moving and breathing, slowly and mindfully, throughout the hour. Since we do use this space for tone-setting, we select appropriate readings for sharing out loud during the final resting posture (and sometimes early in the session for setting intentions), that help frame a mindset conducive to the intensity and soulfulness of the activities that will comprise the coming day.

\section{Yoga for Focusing Attention}

Several years ago one of our students described how yoga was instrumental in her ability to be in the present something that is requisite in showing up in relationship with emotional intelligence. She described herself as having a "wildly active and discursive brain," making it nearly impossible for her to stay focused on what was directly in front of her. For her, one auditory remark or visual expression would lead to a related thought which would then lead to other related thoughts - each removing her further from the here and now. Myriad sensory "branches" sprouted like lightening, making her the quintessential "audience from hell" who can only stay tuned for perhaps the first two words of the first sentence of what might be an important announcement, or an important lecture. She described her struggles with sitting meditation, which she pursued as a means for quieting her mind: "I keep telling my brain to be quiet and be attentive to my breath - over and over again! I'm lucky to go ten seconds without drifting away."

She had a new and revealing experience in the yoga sessions of our EQ course. "In sitting meditation I am never able to just follow my breath - to actually stay with it - for more than a few seconds. But when I had to add physical flow with my breath, as in movement into or out of a posture, or movement from one posture to another, the additional sensory focus that was required kept me in the present - which was a sensation I had rarely experienced." She described our yoga sessions as unique opportunities to be truly present, and to enjoy the rare gift of a focused yet calm mind. She subsequently developed complimentary strategies for quieting her discursive thoughts in other venues where being present and mindful were essential EQ skills, such as in being acutely empathic in a coaching or other active listening space, or in giving or receiving feedback.

\section{Yoga for Increasing Self-Awareness}

What our student described was the attendant focus that can be gained when breath and movement are coordinated. Adding prescribed physical movement to breathing may provide an additional vehicle or anchor for mindful focus, while simultaneously providing a wealth of somatic and sensory input to explore as self-observation. For some, physical or body-based information is central during movement. How does the lower-back feel when moving between an arched-back "cat pose" during inhale to a rounded-back "child's pose" during exhale? With practice, observation and information about breathing also arises. How does the breath respond during challenging movement? During relaxing movement? Attendant emotional and thought-based responses may also arise. What emotions emerge as one stays in pose of a child for multiple breaths? What thoughts arise during physically demanding movement? In short, yoga provides the opportunity to learn about ourselves. We learn about ourselves as we pay attention to what we feel and how we respond. In the EQ curriculum, we use yoga as a vehicle to facilitate the experience of being present with the various ways we respond physically, emotionally and psychologically in any given moment.

\section{Yoga for Nervous System Regulation}

We are all born with a stress response that arises in the presence of perceived danger. Danger can come as physical discomfort or pain, and is often cloaked in negative thoughts and emotions like fear, anger and hurt. 
Using fear as an example, despite what the antecedent of the fear may be, the human nervous system has but one set of innate stress responses emanating from our amygdale: an increase in breath rate, muscle tension, heartbeat and stress hormones like cortisol. In fact, the typical stress response is a combination of all of these.

With increased focus and self-awareness, the real-time stress response based in the nervous system becomes more apparent. Noticing and better understanding the correlation between emotions, thoughts, sensations and, in particular, breath, is the gateway to learning how to employ yoga to regulate the natural stress responses. We hope that honing students' ability to observe bodily and psychological changes will include a growing ability to mitigate the stress response through breathing. Moreover, cultivating a non-judgmental capacity to observe how thinking, feelings and breathing interact gives us more opportunity to intervene with all three. Done in yoga practice (and in EQ class) we experience a laboratory of attention, observation and regulation that we can expand and carry into everyday interactions to give us more control over how we respond to life's challenges.

\section{Yoga for Integration and Processing}

During our closing hour of yoga, scheduled as the final formal activity of the day, we follow a slow yoga format, focused on steady calm breathing. Some of our participants - most of them - are at a place of emotional and relational fatigue at day's end, and may be struggling to process the impact of the day's intensity. Our intention with closing yoga is to coax the system out of any induced stress, helping nurture the counterbalance of the "rest and digest" function of the nervous system. We may select readings for "savasana," the final resting posture, that correlate with the activities and experiences of the day to help bring integration and understanding. As such, our evening yoga sessions strive to achieve some level of mental and emotional peace and quiet, as well as some level of thoughtful contemplation of the changes that each participant is experiencing in awareness and perspectives of self and others. The on-campus ending yoga sessions serve a similar function in helping participants to integrate and process the activities of the day that they just completed.

\section{Significant Outcomes}

We can now confidently offer some concrete observations from our sixteen-year experiment with delivering an experiential program to enhance emotional intelligence skills and sensibilities, as well as concrete observations for the contribution that adding yoga practice has made to our class activities and content. On this last point, we are confident that yoga has enhanced the impact from each of the other key elements of our programming. The critical presence and empathic listening demanded for effective coaching is supported by an enhanced experience of being attentive and mindful in yoga practice. Participants are able to bring more intentional presence, self-awareness and clear communication, based in self-regulation, to their coaching and T-Group sessions. The autobiography exercise benefits from a tone-setting yoga prelude that prepares each participant for a more open and vulnerable space, and the day's closing yoga helps to integrate and process this often intense experience with calm contemplation and reflection. Overall, the powerful container that brings the EQ training to an apex - namely the residential retreat- has been measurably enhanced by each morning's yoga-based framing and tone-setting, as well as the opportunity to integrate and de-escalate at day's end.

The positive outcomes from our EQ course are documented in sixteen years of course evaluations and participant comments that have elevated the experience to its place as the university's most popular graduate course elective. The yoga-infused EQ curriculum positively impacts our student culture, both within the business school and across campus. This is reflected objectively in student action plans, exit interviews and focus group feedback, as well as in participant satisfaction surveys. Indeed, since adding yoga to our program six years ago, the action-plan item mentioned most often in participants' ongoing plans has been yoga practice. Most of our EQ students come into our course with no previous background in yoga; they leave with yoga at the top of their action-plan priorities.

\section{Summary - Yoga Now a Permanent Mainstay of our EQ Course}

Every aspect of our EQ course has been enhanced by the addition of mindful yoga practice interspersed at key intervals throughout our programming. It has given our training a tone-setting assist to insure that students bring more attention and caring to challenging days on retreat and in the Dolomites. It has offered a contemplative space for processing and integrating the activities of long days filled with emotional intensity. Perhaps most importantly, it has given our students a take-away component from their EQ course experience that they can readily implement in their daily schedules. Yoga offers an experiential tool to support our students' efforts to hone attention, self-awareness and regulation--critical to the ongoing EQ journey. Looking back at our many years and many course offerings in emotional intelligence, it is hard for us to imagine our present course without its yoga component. And it is hard to fathom why we waited so long - nearly ten years - to add this important practice to our training. 
Finally, our enthusiasm for sharing and spreading the benefits of emotional intelligence is unbounded. If other academic institutions and programs are interested in giving EQ a try, we would gladly offer our time and our energies to help in that process. We have seen such a dramatic shift in the cultures of our graduate programs that we would do whatever is possible to help bring this transformation to other academic communities. Please don't hesitate to ask for our help.

\section{Appendix 1}

\section{Leading with Emotional Intelligence Course Learning Objectives}

1. To enhance both self- and social-awareness, and develop more effective self-management and relationship-management skills.

2. To develop and hone skills for engaging/calibrating the neural limbic system ("fight or flight" response) to replace impulsive and automatic response patterns with conscious choice patterns.

3. To become more effective communicating with work colleagues at all levels in an organization, and with both current and prospective clients and customers.

4. To learn and develop capacity for effective communication to promote staying in relationship (collegial, social, familial) with authenticity and integrity.

5. To develop strategies and tactics for influencing organizational culture to foster authentic communication among members of the organization.

6. To develop effective skills in providing feedback to colleagues and clients.

7. To develop and practice effective peer and professional coaching skills.

\section{Appendix 2}

List of Experiential Activities Comprising Leading with Emotional Intelligence Course (pre-Yoga) ${ }^{\mathrm{ii}}$

* Defining emotional intelligence and its key components

* Presenting the case for the importance of EQ behaviors

* Assessing participants' EQ attributes

* Creating and refining each participant's EQ self-critique

* Exploring the sources of hard wiring -- an autobiography exercise

* Introducing coaching as both a means to enhance EQ and as an expression of highly-evolved EQ

* Training in coaching skills

* Practicing effective coaching skills, framed around EQ attributes

* Exploring and honing effective communication and listening skills

* Introducing and practicing giving and receiving feedback

* Using a series of interactive, experiential group exercises to practice being open, honest, and aware of feelings and emotions -- to practice staying in the moment and staying connected with others

* Using a series of interactive, experiential group exercises in which to apply new skills, to try out new behaviors, and

to receive real-time coaching and feedback

* Using "skill group" (a variation on the traditional "T Group," or Training

Group) to raise self and social awareness, and to practice being present in an unstructured group dynamic

* Using "skill group" to raise participants' authenticity

* Using structured feedback exercises to raise awareness

* Putting together an action plan, based upon a heightened awareness of EQ challenges evolving from all of the course experiences -- an action plan that addresses those patterns, habits and hard-wired behaviors that participants want to change

\section{Appendix 3}

\section{Description of EQ Training Modalities: Coaching, Autobiography, T Group}

\section{Coaching Module}

The first day of our EQ training regimen features a primer on coaching, which includes a written introduction to the art of coaching, a live demonstration of effective coaching technique, and an off-site practice assignment where students help each other hone their active listening and "coaching space" creation skill sets. From the outset we hold our professional graduate students capable of achieving and applying appropriate skills in the service of helping each other move forward in their lives. Our graduate students are accomplished professionals (aerospace and software engineers, project managers, biomedical researchers, student development professionals, etc.) who possess the intellectual heft to 
take on the challenges of effective coaching - ironically, to take on the precise interpersonal skills that, undeveloped, may have been a deterrent to the career growth of many of our students. We are able to bring our participants to an impressive level of coaching finesse in a short time. The off-site simulations surprise all participants in the depth of insights that emanate from the exercise. Students report that they take their newly-formed active listening skills to their work relationships with notably positive results. In our written coaching primer that we give to each student, we summarize the important connection between EQ behaviors and effective coaching:

"No single professional service more completely captures the elements of emotional intelligence (EQ) than does effective coaching. Coaching is both a means for enhancing EQ awareness and behaviors, as well as an expression of emotional intelligence in practice. Hence, one of the key sets of skills to develop and hone as part of a program for enhancing emotional intelligence is the set of skills that comprise the effective coach." ${ }^{\text {iii }}$

\section{Autobiography Exercise}

The autobiography exercise is one of the most powerful experiences in our EQ curriculum. Participants write fourpage abridgments of their life stories. We encourage everyone to be as transparent as they feel comfortable being, noting that they are substantially formed in life by the challenges they have faced, by the peaks and valleys of their pathways, and by their struggles and resilience. This invites a look at parts of their lives they rarely share and, in many cases, rarely consciously revisit. ${ }^{\text {iv }}$

The first day of our three-day residential retreat is devoted to sharing autobiographies in a small group. Participants spend six to eight hours with members of their "T Group," comprising five to seven individuals, with whom students have spent most of the quarter's preceding classroom exercises. Each person shares his or her story via narration with this small group of colleagues, with approximately an hour designated for group inquiry and discussion focused on understanding and acknowledging the experiences (both past and in the moment) of the member who shares. By nightfall on the first retreat day, the mood has become noticeably subdued, contemplative, and, for many, peaceful through catharsis.

In our formal debriefing of the day's activities people speak to the impact of sharing and hearing personal stories. In particular, they speak to an empathic understanding of their own and others' resilience. Listening to their colleagues' share their personal stories awakens new perspectives on the complexity, resilience and multifaceted depth of the human experience. The experience diminishesfirst impressions and reductive stereotypes that are based on limited understanding of, and appreciation for, one's own connection with others' life experiences. In the same vein, sharing one's own story delivers a vulnerable yet supported experience of feeling non-judgmentally heard and accepted, even upon sharing aspects of one's self heretofore perceived as shameful, unacceptable, and un-sharable. The experience often further liberates individuals to be more open and truthful in subsequent T-Group interactions.

\section{T-Group Practice}

A key learning modality throughout the emotional intelligence course is the traditional T Group (Training Group). TGroup training creates a unifying communication framework that draws students closer to each other with experiences of sharing and communion. The $\mathrm{T}$ Group helps build visceral connections among participants, drawing them together with a willing openness and a skill set that enhances trust, empathy, personal safety, intimacy, caring, curiosity, and mutual respect. A full description of the T-Group methodology and its application to emotional intelligence training is beyond the scope of this appendix. See the reference in this footnote for a detailed explanation of T Groups. ${ }^{\mathrm{V}}$

We have been using the T Group in our EQ programming from the outset and believe it is instrumental in creating the cross-campus popularity of the course, as well as being a unifying force for all graduate students at Seattle University. Preconceptions that traditionally divide business students (the "Gordon Gekko" vi stories) from law students (the "greedy corporate lawyers" stories) from fine arts students (the "dramatic, emotive and eccentric" stories) melt away under the artfully directed regimen of the T Group. We believe our EQ programming, particularly the dynamics of the T Group, helps diverse graduate students see each other as whole people.

\footnotetext{
${ }^{\mathrm{i}}$ For more information about our annual EQ course in the Italian Dolomites, as well as about other opportunities for EQ training, see www.pauseconnect.com. Pause, Inc. assists Seattle University in the delivery of the Dolomites program.

ii Taken from Weis, W.L. \& Arnesen, D. (2007). "Because EQ Can't Be Told": Doing Something about Emotional Intelligence. Journal of Organizational Culture, Communications and Conflict, 11(2), 113-123.

iii Included in Weis, W. L. (2006) Unpacking the Experience: A Primer on Coaching. Seattle: Teams and Leaders, Inc.
} 
${ }^{\text {iv }}$ For a complete discussion of the autobiography exercise and on its impact on our EQ students, see Alexandre, N. \& Weis, W.L. (2017). Weltanschauung and the Autobiography Exercise: Transforming the Learning Experience by Sharing Personal Stories. Journal of Jesuit Business Education. Summer 2018..

${ }^{\mathrm{v}}$ For a thorough discussion of the T Group, including its history since 1947 and its direct application to EQ training, see Weis, W. L., Arnesen, D., \& Hanson, L. (2009). The Use of Training Groups (T-Groups) in Raising Self and Social Awareness and Enhancing Emotionally Intelligent Behaviors. Journal of Organizational Culture, Communications and Conflict, 13(2), 83-104.

${ }^{v i}$ The anti-hero from the movie Wall Street (1987). Directed by Oliver Stone. Distributed by $20^{\text {th }}$ Century Fox (126 minutes). 